\title{
Investigation of Grain Exchange Interaction Effects on The Magnetic Properties of Strontium Hexaferrite Magnets
}

\section{Novrita Idayanti1,2, Dedi², and Azwar Manaf ${ }^{1}$}

${ }^{1}$ University of Indonesia, Faculty of Mathematics and Natural Science, Physic Department, Kampus UI, Depok, 16424, Indonesia

${ }^{2}$ Research center for electronics and telecommunications Indonesian Institute of Sciences, Kampus LIPI JI. Sangkuriang, Bandung, 40135, Indonesia

\section{Abstract}

Results of investigation of grain exchange interaction effects on the magnetic properties of strontium hexaferrite $\left(\mathrm{SrO} .6 \mathrm{Fe}_{2} \mathrm{O}_{3}\right)$ based samples are reported. The investigation was subject to the evaluation of magnetic properties of three samples prepared in three different routes. Sample A was prepared through the refinement of mechanically alloyed powders. Sample B was obtained from the mechanically alloyed of sample

Corresponding Author:

Azwar Manaf

azwar@sci.ac.ui.id

Received: 19 February 2019

Accepted: 5 March 2019

Published: 16 April 2019

Publishing services provided by

Knowledge E

(c) Novrita Idayanti et al. This article is distributed under the terms of the Creative Commons

Attribution License, which

permits unrestricted use and redistribution provided that the

original author and source are credited.

Selection and Peer-review under the responsibility of the ICBSA Conference Committee.

\section{G OPEN ACCESS} $A$ and subsequently irradiated ultrasonically. Whereas the sample $C$ was obtained through mechanically alloyed powder of sample $A$ with the addition of $15 \%$ poly vinyl alcohol (PVA) as the non-magnetic binding agent. Subject to phase identification and evaluation of $x$-ray diffraction data for sample $A$ and $B$, it was found that both samples are fully crystalline single-phase materials with mean crystallite size respectively 25 $\mathrm{nm}$ and $13 \mathrm{~nm}$. Both samples have indicated a high value of $\mathrm{M}_{r} / \mathrm{M}_{s}$ ratio subject to evaluation based on comparison between the mean crystallite size and the exchange length values. Additionally, $\mathrm{M}_{r} / \mathrm{M}_{s}$ value was calculated from the $\mathrm{M}_{r}$ of a minor loop and $\mathrm{M}_{s}$ of theoretical value which resulted in a ratio about 0.5 . The addition of a non-magnetic binder in the SHF sample reduces the coercivity significantly due to magnetostatics effect and remanence due to less mass fraction of magnetic phase in the sample.

Keywords: Magnetic properties; hard ferrite; nanocrystalline; grain exchange interaction; permanent magnets.

\section{Introduction}

Strontium hexaferrite with the chemical formula $\mathrm{SrO} .6 \mathrm{Fe}_{2} \mathrm{O}_{3}$ is well known hard magnetic phase for permanent magnets [1], which still dominate the application in various sectors like electric and electronics, telecommunication, magnetic recording media, and microwave devices [2]. The SHF combines several physical characteristics like high values of magnetocrystalline constant $\left(K=3.6 \times 10^{6} \mathrm{erg} . \mathrm{cm}^{-3} \mathrm{MJ} / \mathrm{m}^{3}\right)$, saturation 
magnetization $\left(\mathrm{J}_{s}=70 \mathrm{emu} / \mathrm{g} \mathrm{T}\right)[3]$, curie temperature $\left(\mathrm{T}_{c}=737^{\circ} \mathrm{K} / 463^{\circ} \mathrm{C}\right)[4,5]$ which are mostly required for permanent pagebreak magnets. The SHF is a ceramic based compound with excellent chemical stability and high corrosion resistance making SHF most suitable for permanent magnets. In addition, the raw material components for SHF like $\mathrm{Fe}$ and $\mathrm{Sr}$ are those abundantly available on earth has lead the SHF as the cheap and low costs magnets.

Generally, SHF is prepared by conventional powder metallurgy or sintering method $[6,7]$ which involves mixing iron oxide $\left(\mathrm{Fe}_{2} \mathrm{O}_{3}\right)$, strontium carbonate $\left(\mathrm{SrCO}_{3}\right)$ and additives. After mixing and pelletizing, the sintering is applied at temperature $1373 \mathrm{~K}-$ $1573 \mathrm{~K}[8,9]$ to facilitate the solid state reaction toward the hard magnetic phase formation [10]. Several non-conventional techniques, i.e. sol gel [11], citrate-gel process [12], co-precipitation [13], self-flash combustion [14], and mechano chemical process [15], injection molding [16] have also been developed in various laboratories to prepare the SHF and improve the magnetic properties. However, among the all processes developed in the laboratory scale, the conventional powder metallurgy has been used for commercial production.

The magnetic properties of permanent magnets is microstructure sensitive for which the grain or crystallite size, crystal shape and phase existence determines the coercivity, remanence and maximum energy product, $(\mathrm{BH})_{\max }$ [17]. The high coercivity can be obtained if the mean crystallite size within the size of single domain particle [18]. The size of single domain particle of SHF based on theoretical calculation is $0.74 \mu \mathrm{m} \sim 740$ $\mathrm{nm}$ [19]. Hence, the mean crystallite size of SHF should be controlled during wet or dry milling to obtain powder of narrow particle size distribution. The mean crystallite size of SHF should be in the range $700 \mathrm{~nm}-900 \mathrm{~nm}$, and the crystallite growth during sintering for densification has to be avoided for achieving good magnetic properties. When the crystallite size of magnetic materials is much smaller than that of single domain particle size, effect of grain exchange interaction can take place [20]. However, when the crystallite size is extremely small, effect of superparamagnetic can take place [18]. Several authors have reported that the grain exchange interaction affected the remanence and coercivity [21]. The remanence is enhanced with the expense of coercivity [22]. It was also shown that the effect of exchange coupling between grains increased due to the existence of amorphous grain boundary [23]. The existence of nonmagnetic grain boundary phase reduces the exchange-coupling interaction and decreases remanence and coercivity. Remanence enhancement is due to magnetic moments interaction of adjacent grain as long as the exchange length is relatively long to contribute the increase in surface to volume ratio of bulk magnets. The coercivity 
of exchanged grains is influenced by density of grain boundary, which results from the variations of magneto crystalline anisotropy constant, K1. Theoretically, the anisotropy constant at exchange grain interaction areas is reduced to $K 1 / N^{1 / 2}$, where $N$ is the average number of neighbor grains. While, the anisotropy constant of the inner part of the grains still unchanged. Both of them regard the anisotropy of the coupled part of the grain as a fixed constant smaller than K1 [24]. The strength of the grain interaction in magnetic materials can be analyzed by measuring the remanence ratio.

In this study, we carried out the investigation on the effect of grain interaction effect to the magnetic properties of SHF based magnets by means of experimental simulation. The single domain particle of the sample is prepared by mechanical alloy and subsequent ultrasonic irradiation of high frequency. The ultrasonic treated SHF particles are decoupling through the addition 15\% of Poli Vinyl Alcohol (PVA) so that inter-grain interactions are blocked. The effect of grain interactions on their magnetic properties with no exchange grain interaction is compared.

\section{Experimental Method}

The experiment procedures in this research are devided in three stages; shyntesize SHF by mechanical alloying, ultrasonic, and preparation of bonded SHF with addition 15\% PVA.

\subsection{Stage 1: Preparation of SHF samples}

The stoichiometric amounts of $\mathrm{Fe}_{2} \mathrm{O}_{3}$ and $\mathrm{SrCO}_{3}$ precursors with a purity level greater than $99 \%$ were mixed in vials containing hardened stainless-steel balls of a planetary ball milling apparatus. The mass ratio between precursors and the ball was fixed 1:10. The ball-milling process was carried out in alcohol dispersion media under a rotating speed of $1500 \mathrm{rpm}$ for 6 hours. The mechanically alloyed powders were dried and compacted in a cylindrical die subject to a uniaxial pressure of $75 \mathrm{~kg} / \mathrm{cm}^{2}$. The green compact was subsequently calcined at a temperature $1200{ }^{\circ} \mathrm{C}$ for 3 hours lead to fully crystalline samples. The crystalline sample was then re-milled together with $\mathrm{SiO}_{2}$ and $\mathrm{CaO}$ additives for 16 hrs. 3.5 grams of fine crystalline powders were compacted at a load of 5 tons by a hydraulic press machine in a round shape, and then were sintered at a temperature $1250^{\circ} \mathrm{C}$ for 1 hour. Sample that prepared from this stage is called sample

A. Other powders will be a feedstock of ultrasonic irradiation treatment. 


\subsection{Stage 2: Preparation of crystalline SHF powder subject to ultrasonic irradiation treatment}

SHF powders were poured into $300 \mathrm{ml}$ distillate water together with 3 drops of ethylene glycol in a beaker glass. A schematic of ultra-sonification set up is illustrated schematically in Fig. 1.

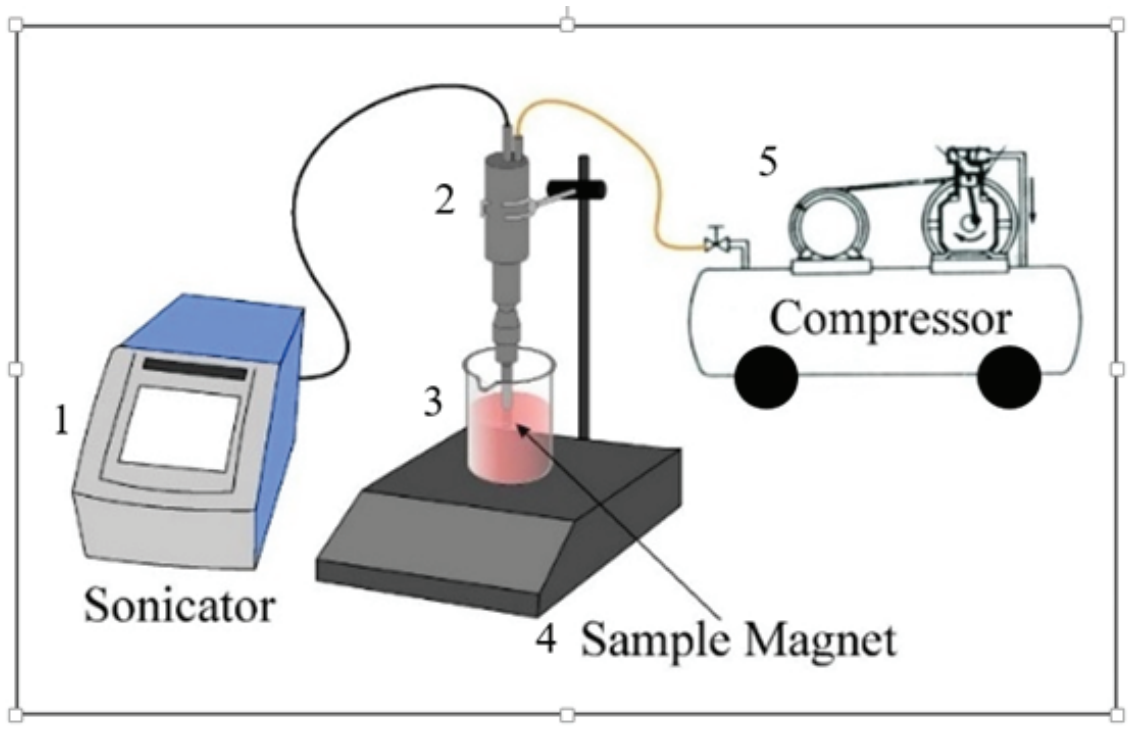

Figure 1: A schematic of sonification set-up, legends; 1. sonicator of $20 \mathrm{kHz}$ frequency; 2. ultrasonic horn; 3. Beaker glass; 4. Crystalline magnet powder in dispersed media and 5. compressor.

The ultrasonic irradiation process lasted for 10 hours under the following operating condition: the horn amplitude was $50 \mathrm{~dB}$ with a pulse on for $10 \mathrm{sec}$ and pulse off for $5 \mathrm{sec}$ at a $20 \mathrm{kHz}$ automatic frequency tuning. The total energy consumed was $\sim 5 \mathrm{~kJ}$. The temperature of the reaction process was monitored using by a digital temperature probe control. The sonicator used is Qsonica Q 700 with a $12 \mathrm{~mm}$ probe diameter. Magnetic powders obtained after ultrasonic irradiation treatment were dried and then compacted and sintered. Sample that prepared in this stage is sample B.

\subsection{Stage 3: Preparation of bonded SHF samples}

$15 \%$ of binder PVA were disolved in distillate water and mixed with calcined SHF powders. Both ingredients were then stirred until well blended, then the mixtures were dried. The dried powder was then compacted under a load of 5 tons and cured at a temperature $250^{\circ} \mathrm{C}$ for 1 hour. Sample that prepared from this stage is called sample C. 


\subsection{Samples characterization}

Crystalline powder of SHF were analyzed by a Bruker D8 series x-ray diffractometer (XRD) using $\mathrm{Cu} \mathrm{K} \alpha\left(\lambda=1.5418 \AA\right.$ ) radiation in $2 \theta$ ranging from $20^{\circ}$ to $70^{\circ}$. The microstructure of the fracture surface of each sample was observed under a JEOL-JSM IT-300 scanning electron microscope (SEM). The magnetic properties were evaluated by a Magnet Physik Permagraph. Determination of mean crystallite size was evaluated from Williamson-Hall plot (W-H plot) [25]. All un-overlapping peaks of high diffraction angle were taken into account for a $\mathrm{W}-\mathrm{H}$ plot.

\section{Results and Discussion}

\subsection{Phase study and mean crystallite size determintaion}

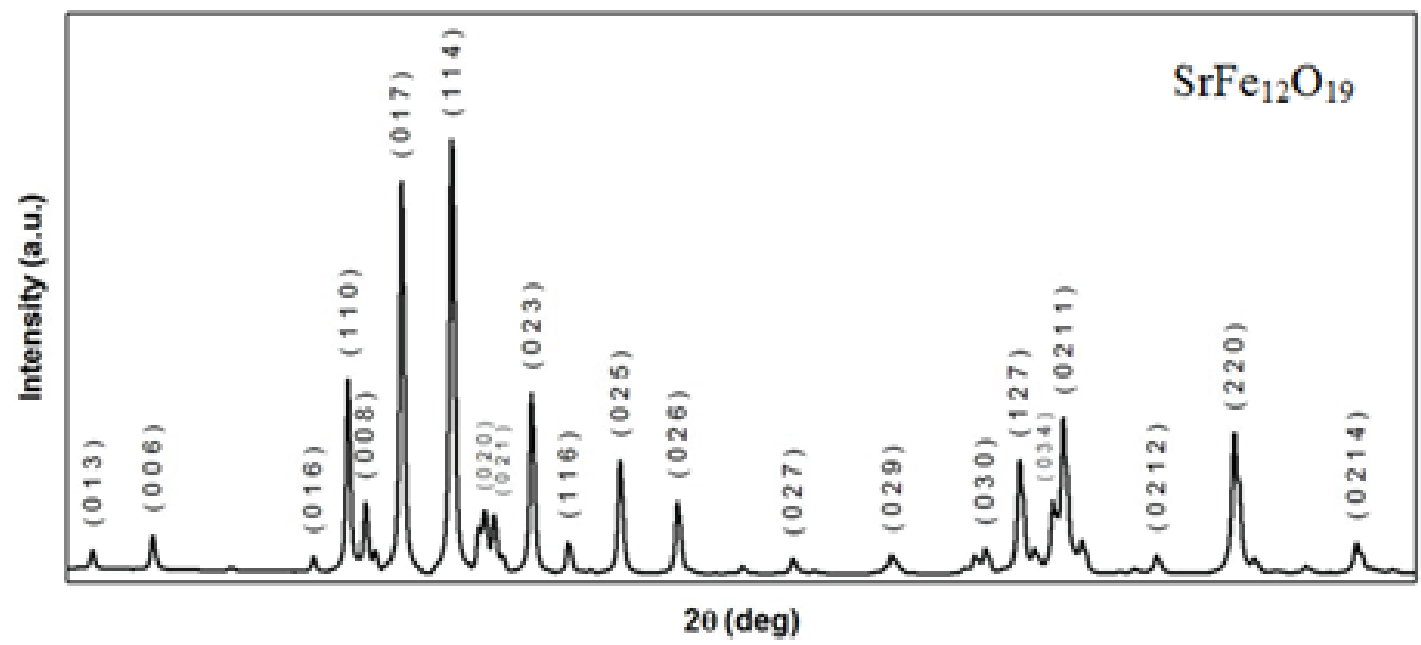

Figure 2: XRD curve of $\mathrm{SrO} .6 \mathrm{Fe}_{2} \mathrm{O}_{3}$ synthesized by mecahnical milling method.

Fig. 2 shows a plot of $x$-ray diffraction pattern of a sample $A$ and that of samples $B$ in Fig. 3. All diffraction peaks of XRD patterns in the two figures were successfully identified and indexed as the phase constitution sample materials. The patterns in Fig. 2 and 3 are in a good agreement with the hexagonal structure of M-type $\mathrm{SrO}^{2} 6 \mathrm{Fe}_{2} \mathrm{O}_{3}$ referred to ICSD Ref. code. 98-004-3603 (PANalytical' X'Pert HighScore Report). It is confirmed that both samples $A$ and $B$ is a single-phase material with $\mathrm{SrO}^{-6} \mathrm{Fe}_{2} \mathrm{O}_{3}$ the only phase present.

The mean crystallite size which evaluated from the $\mathrm{W}-\mathrm{H}$ plots of the two samples has resulted in sizes of $25 \mathrm{~nm}$ for sample $A$ and $13 \mathrm{~nm}$ for sample B. Hence, the two samples consisted of nanocrystalline grains with only slightly different in mean 


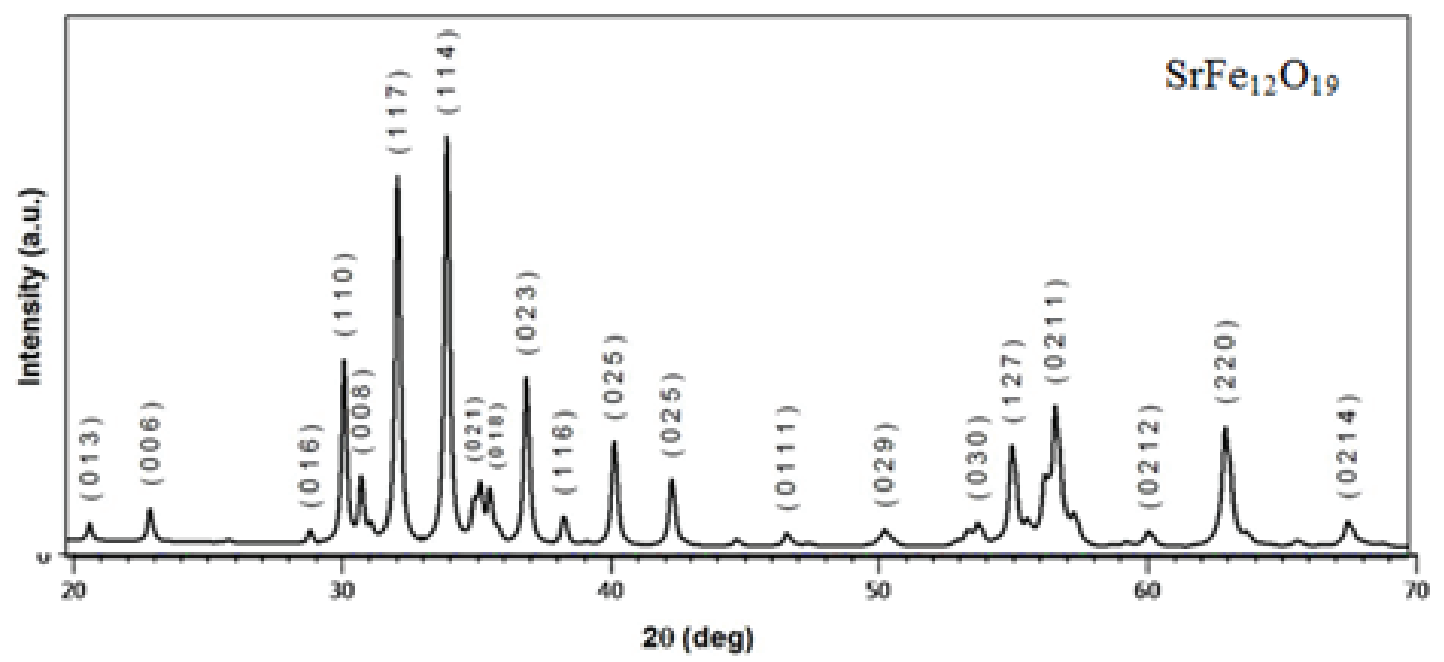

Figure 3: XRD curve of $\mathrm{SrO} .6 \mathrm{Fe}_{2} \mathrm{O}_{3}$ synthesized by ultrasonic process.

crystallite size. The ultrasonic treatment has succeeded reducing the mean crystallite size of mechanically alloyed powder. When the ultrasonic irradiation is long enough, the monocrystalline particles could be obtained [26]. Palomino has reported that monocrystalline particles of SHF can be obtained after ultrasonic irradiation process for 1 hour [27]. It is worthy to note that sintering at a high temperature for samples $A$ and $B$, the mean crystallite size is remaining small still fall in the nanometer regime. Hence, there has been no crystallites growth taken place in both samples due to high temperature sintering treatment. Samples of magnetic crystals with such ultra-fine size in the nanometer regime must be undergo grain exchange interaction effects. For SHF, the exchange length is $40 \mathrm{~nm}$ [28] which larger than that of mean diameter of magnetic crystals in the sample A and sample B.

\subsection{Microstructures observation}

Micrograph that shown in Fig. 4 represents the fracture surface of (a) sample A, (b) sample B and (c) sample C. The image shows in Fig. 4a and 4b are clusters of crystallite instead of the crystallites itself since the crystallite size referring to results of $\mathrm{W}-\mathrm{H}$ plots is much finer than those visually seen in micrographs of Fig. 4. Visually, micrograph that showed in Fig. 4b is much compact and clusters appear much more crowded when compared with that of shown in Fig. 4a. Effect of ultrasonic treatment to the crystalline powder has improved the connectivity among the clusters. Referring to mass density evaluation, the density of samples $A$ and $B$ is respectively $4.73 \mathrm{gr} / \mathrm{cc}$ and $5.00 \mathrm{gr} / \mathrm{cc}$. Hence, better cluster connectivity was obtained after ultrasonic treatment. In contrary, 
the micrograph in Fig. 4c appears in a very much different morphology in which grains and clusters of grain are already coated by a binder material. The magnetic clusters must be already separated by a nonmagnetic binder. Which such microstructure there is no grain exchange interaction effects might have occurred effectively in the sample C.

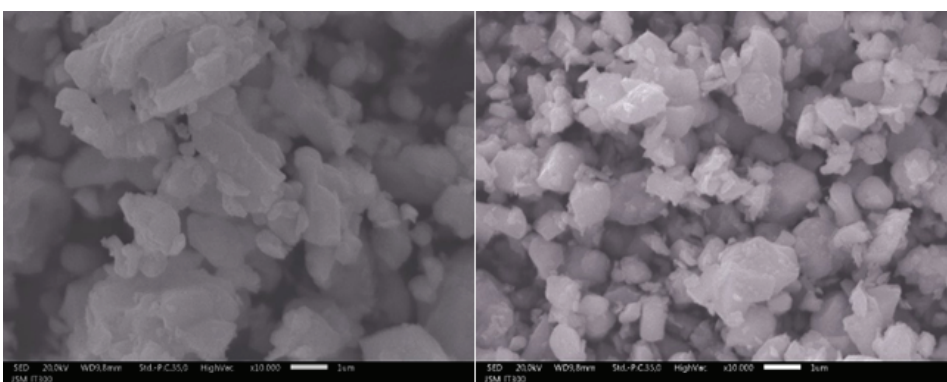

(a)

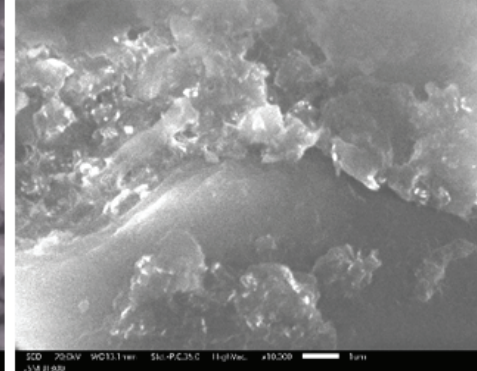

(c)

Figure 4: SEM images of $\mathrm{SrO} 6 \mathrm{Fe}_{2} \mathrm{O}_{3}$ (a) sample $\mathrm{A}$ by mechanical alloying method (b) sample $\mathrm{B}$ by ultrasonic process, and (c)sample C with PVA 15\%.

Sample $C$ does not show a clear grain of magnetic material because the PVA content covers the grain boundaries of each material. This condition causes an incomplete grain exchange interactions in sample $\mathrm{C}$. The explanation of the occurrence of interactions at the grain boundary and which did not occur in Fig. 4 is illustrated in Fig. 5 (a) and 5 (b).

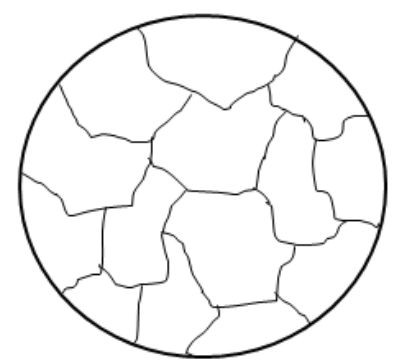

(a)

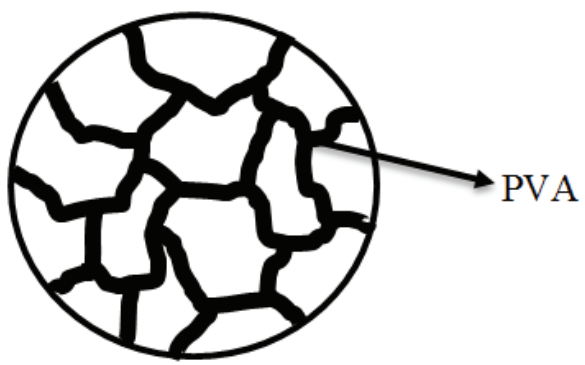

(b)

Figure 5: Grain Boundaries of $\mathrm{SrO} 6 \mathrm{Fe}_{2} \mathrm{O}_{3}$ (a) without PVA (b) with PVA 15\%.

When two grains of magnetic material are in direct contact each with other, the magnetic moment on the grain surface with different orientations will produce the exchange coupling interaction that aligns direction of the magnetic moment on the grain surface continuously. The magnetic moment becomes parallel, so remanence magnetization increases. The results of this research show that when adding nonmagnetic material (PVA) the interaction of grain changes will not occur. Magnetic properties decrease because interactions between grain are blocked by non-magnetic material. This is to prove that if an interaction occurs then the magnetic properties 
will increase. beside that, coupling exchange interaction in magnetic materials are also influenced by grain size. If the grain size is reduced to a nanometer, the magnetic properties will obviously change. The changes in the magnetic properties of the three samples will be discussed in the analysis of magnetic properties.

\subsection{Magnetic properties}

Fig. 6 compares the $(\mathrm{M}-\mathrm{H})$ loops for samples A, B and C. Some magnetic properties derived from the $\mathrm{M}-\mathrm{H}$ loops were summarized in Table 1. The term "magnetization at maximum field, M" implies that the quoted value is the magnetization value at a magnetizing field $12 \mathrm{kOe}$. The quoted $\mathrm{M}$ value in Table 1 must be very much lower than the saturation magnetization, $\mathrm{M}_{s}$ value of SHF $74.3 \mathrm{emu} / \mathrm{gr}$ [29]. The $\mathrm{M}-\mathrm{H}$ loop of sample $A$ and $B$ looks similar with values of remanence and coercivity for the two samples almost identical. The $\mathrm{M}_{r} / \mathrm{M}_{s}$ ratio of SHF listed in Table 1 was calculated subject to the theoretical $M_{s}$ value of SHF. The fact that the $M_{r} / M_{s}$ for SHF samples do not exceed the theoretical limit of 0.5 for non-interacting, randomly oriented unaxial easy axis particles [30]. Hence, there is no grain interacting effect occurred in samples A and B. However, consider that the mean crystallite size of sample A and B is respectively $25 \mathrm{~nm}$ and 13 $\mathrm{nm}$, which both values less than the exchange length of SHF $40 \mathrm{~nm}$, then the effect of grain exchange interaction [31] must be taken place in the SHF samples. The current $M_{r} / M_{s}$ value of $<0.5$ for SHF samples might indicate that $(M-H)$ loops as plotted in Fig. 6 for samples $A$ and $B$ represent the minor loops. In addition, the mass density of the two samples as listed in Table 1 is still lower than $5 \mathrm{~g} / \mathrm{cc}$ the theoretical mass density of SHF [32]. It is believed that with such $M_{r} / M_{s}$ ratio values that evaluated from $M_{r}$ of a minor loop and theoretical $M_{s}$ value for SHF, the exchange interaction effects took place in the samples $A$ and $B$.

The $(\mathrm{M}-\mathrm{H})$ loop of sample $\mathrm{C}$ has a loop with coercivity and remanence values are respectively low when compared with those samples A and B. Obviously, the low remanence value of sample $C$ is associated with a low mass density value due to the presence of a non-magnetic binder in the sample. With $15 \%$ mass fraction of the nonmagnetic binder of the sample, only $85 \%$ the mass of the sample in the whole volume contributes to the magnetization. Consequently, the $\mathrm{M}_{r} / \mathrm{M}_{s}$ value for bonded SHF sample is only 0.29 . The low coercivity value of sample $C$ might be due to magnetostatics effect arising from the presence of significant number of non-magnetic material inside. 


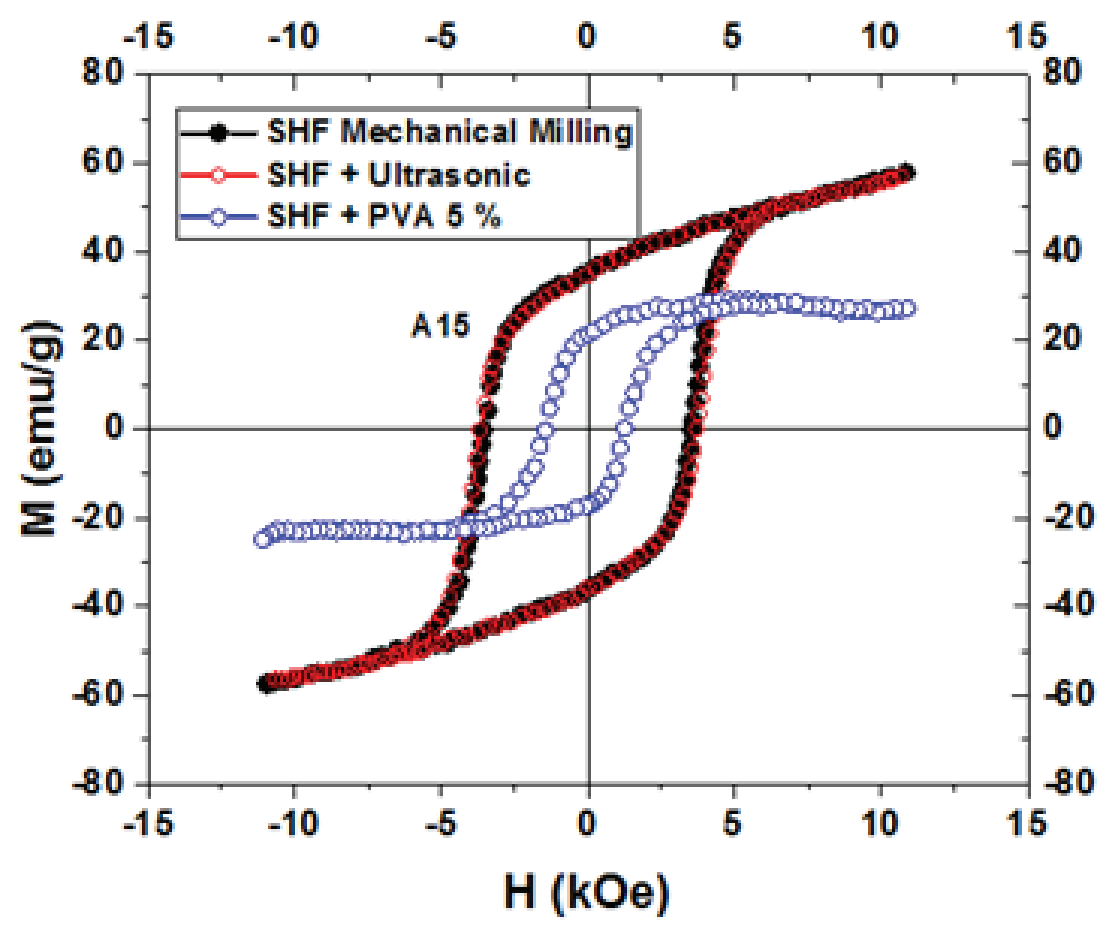

Figure 6: The $(\mathrm{M}-\mathrm{H})$ loops of samples $\mathrm{A}, \mathrm{B}$ and $\mathrm{C}$.

TABLE 1: Magnetic properties and massdensity SHF samples.

\begin{tabular}{|c|c|c|c|c|c} 
Sample & $\begin{array}{c}\text { Magnetization } \\
\text { at a maximum } \\
\text { field M (emu/g) }\end{array}$ & $\begin{array}{c}\text { Magnetization } \\
\text { remanence } M_{r} \\
(\mathrm{e} \text { (emu/g) }\end{array}$ & $\begin{array}{c}\text { Coercive field } \\
H_{c}(k O e)\end{array}$ & $M_{r} / M_{s}$ ratio & $\begin{array}{c}\text { Mass density } \rho \\
\text { (g/cc) }\end{array}$ \\
\hline A & 57.231 & 36.190 & 3.497 & 0.49 & 4.70 \\
\hline B & 57.325 & 36.146 & 3.704 & 0.49 & 5.00 \\
\hline C & 29.045 & 21.528 & 1.667 & 0.29 & 2.33 \\
\hline
\end{tabular}

\section{Conclusion}

Investigation on the effect of grain exchange interaction in the SHF sample has been made by comparing the magnetic properties derived from the hysteresis loops. SHF sample of mechanically alloyed has mean crystallite size $25 \mathrm{~nm}$. While mean crystallite size of $13 \mathrm{~nm}$ was obtained in the sample prepared through mechanical alloying combined with ultrasonic irradiation. Both samples have indicated a high value of $M_{r} / M_{s}$ ratio subject to evaluation based on comparison between mean crystallite size and the exchange length values. Additionally, $\mathrm{M}_{r} / \mathrm{M}_{s}$ value was calculated from the $\mathrm{M}_{r}$ of a minor loop and $\mathrm{M}_{s}$ of theoretical value which resulted in a ratio about 0.5 . The addition of non-magnetic binder in the SHF sample reduces the coercivity significantly due to magnetostatics effect and remanence due to less mass fraction of magnetic phase in the sample. 


\section{Acknowlegment}

The authors gratefully acknowledge the Ministry of Research, Technology and Higher Education Indonesia for providing support and funding under program Insinas 2018. Thanks also for the Physics Department University of Indonesia and the research center for electronics and telecommunications-LIPI.

\section{References}

[1] A. Cochardt, "Modified Strontium Ferrite, a New Permanent Magnet Material," vol. 1273, pp. 1-3, 1963.

[2] M. Jamalian, "Journal of Magnetism and Magnetic Materials An investigation of structural, magnetic and microwave properties of strontium hexaferrite nanoparticles prepared by a sol - gel process with doping SN and Tb," J. Magn. Magn. Mater., vol. 378, pp. 217-220, 2015.

[3] A. E. Sleptsova, I. V Roslyakov, E. S. Kozlyakova, A. V Vasiliev, R. E. Dinnebier, and P. E. Kazin, “with giant coercivity," pp. 479-482, 2018.

[4] Z. F. Zi, Y. P. Sun, X. B. Zhu, Z. R. Yang, J. M, and W. H. Song, “Journal of Magnetism and Magnetic Materials Structural and magnetic properties of SrFe12O19 hexaferrite synthesized by a modified chemical co-precipitation method," vol. 320, pp. 27462751, 2008.

[5] B. Abraime et al., “Author 's Accepted Manuscript," Ceram. Int., 2017.

[6] Z. Jin, W. Tang, J. Zhang, H. Lin, and Y. Du, "Magnetic properties of isotropic SrFe12019 fine particles prepared by mechanical alloying," vol. 182, pp. 231-237, 1998.

[7] R. C. Pullar, "Hexagonal ferrites: A review of the synthesis, properties and applications of hexaferrite ceramics," in Progress in Materials Science, 2012, vol. 57, no. 7, pp. 1191-1334.

[8] A. Xia, C. Zuo, L. Chen, C. Jin, and Y. Lv, “Journal of Magnetism and Magnetic Materials Hexagonal SrFe12O19 ferrites: Hydrothermal synthesis and their sintering properties," J. Magn. Magn. Mater., vol. 332, pp. 186-191, 2013.

[9] R. S. A. N. Daud, "Preparation and Characterization of Sr1-xNdxFe12O19 Derived From SteelWaste Product via Mechanical Alloying.pdf.".

[10] J. Luo, "Preparation of Strontium Ferrite Powders by Mechanochemical Process Juhua Luo," vol. 116, pp. 1736-1740, 2012. 
[11] Q. Fang, Y. Liu, P. Yin, and X. Li, "Magnetic properties and formation of Sr ferrite nanoparticle and Zn, Ti / Ir substituted phases," vol. 234, pp. 366-370, 2001.

[12] C. Rong, J. P. Liu, C. Rong, and J. P. Liu, "Grain boundary contribution to recoil loop openness of exchange-coupled nanocrystalline magnets Grain boundary contribution to recoil loop openness of exchange-coupled nanocrystalline magnets," vol. 172510, no. 2009, pp. 92-95, 2012.

[13] Y. Sun, R. Gao, and W. Feng, "Effects of intergranular phase on the exchangecoupling interaction and effective anisotropy of nanocrystalline Nd2Fe14B materials," vol. 140, pp. 112-115, 2006.

[14] P. M. Davis and M. E. Evans, "Interacting Single-Domain Properties of Magnetite Intergrowths," vol. 81, no. 5, pp. 989-994, 1976.

[15] S. Cisowski, "Interacting vs. non-interacting single domain behavior in natural and synthetic samples," vol. 26, pp. 56-62, 1981.

[16] D. Kechrakos and K. N. Trohidou, "Magnetic properties of dipolar interacting singledomain particles," vol. 58, no. 18, pp. 169-177, 1998.

[17] C. Fang, R. Metselaar, and G. De With, "Magnetic and electronic properties of strontium hexaferrite SrFe12019 from first-principles calculations Magnetic and electronic properties of strontium hexaferrite SrFe12019 from first-principles," no. May 2014, 2003.

[18] Q. Li, C. W. Kartikowati, S. Horie, T. Ogi, T. Iwaki, and K. Okuyama, "Correlation between particle size / domain structure and magnetic properties of highly crystalline Fe 3 O 4 nanoparticles," Sci. Rep., no. July, pp. 1-4, 2017.

[19] J. Park, Y. Hong, W. Lee, S. An, and J. Seo, "Coercivity of SrFe12O19 Hexaferrite Platelets Near Single Domain Size Hard Magnetic Materials Coercivity of SrFe12O19 Hexaferrite Platelets Near Single Domain Size," no. September, 2015.

[20] H. Endo, Y. Uesaka, Y. Nakatani, N. Hayashi, and H. Fukushima, "Effects of exchange interaction and grain size distribution on cluster size in perpendicular media," vol. 276, pp. 711-712, 2004.

[21] H. Fukunaga, J. Kuma, and Y. Kanai, "Effect of Strength of Intergrain Exchange Interaction on Magnetic Properties of Nanocomposite Magnets," vol. 35, no. 5, pp. 3235-3240, 1999.

[22] A. Poorbafrani, H. Salamati, and P. Kameli, "Exchange spring behavior in Co0.6Zn0.4Fe2O4/SrFe10.5016.75 nanocomposites," Ceram. Int., vol. 41, no. 1, pp. 1603-1608, 2015. 
[23] G. A. O. Ruwei, F. Weicun, C. Wei, W. Biao, H. A. N. Guangbing, and Z. Peng, "Exchange-coupling interaction and effective anisotropy in nanocomposite permanent materials," vol. 47, no. 14, 2002.

[24] H. Search, C. Journals, A. Contact, and M. lopscience, "Exchange interaction through amorphous intergranular layers in a two-phase system Exchange interaction through amorphous intergranular layers in a two-phase system," vol. 3255.

[25] S. Kumar, V. D. Mote, R. Prakash, and V. Kumar, "X-ray Analysis of a $-\mathrm{Al}_{2} \mathrm{O}_{3}$ Particles by," no. January 2017, pp. 1-6, 2016.

[26] X. Pan, $\mathbb{E}$ E. J. Qiu, and $\overparen{A}$. M. Gu, "Preparation and microwave absorption properties of nanosized Ni / SrFe12019 magnetic powder," pp. 2086-2089, 2007.

[27] R. L. Palomino, A. M. B. Miró, F. N. Tenorio, F. S. De Jesús, C. A. C. Escobedo, and S. Ammar, "Ultrasonics Sonochemistry Sonochemical assisted synthesis of SrFe12O19 nanoparticles," Ultrason. - Sonochemistry, vol. 29, pp. 470-475, 2016.

[28] F. Song, X. Shen, M. Liu, and J. Xiang, "One-Dimensional SrFe12O19/NiO.5Zn0.5Fe2O4 Composite Ferrite Nanofibers and Enhancement Magnetic Property," J. Nanosci. Nanotechnol., vol. 11, no. 8, pp. 6979-6985, 2011.

[29] A. P. I. Liai, T. O. Thin, F. Metai, and L. I. C. Redia, "2398 leee Transactions on Magnetics, Vol. Mag-23, No. 5, September 1987 Weaii-lii;Sistant," vol. M, no. 5, pp. 2398-2400, 1987.

[30] E. P. W. E.C. Stoner, "A Mechanism of Magnetism Hysteresis in Heterogeneous Alloys," vol. 826, no. May, 1948.

[31] Y. F. Xu, Y. Q. Ma, S. T. Xu, F. L. Zan, G. H. Zheng, and Z. X. Dai, “Effects of vacancy and exchange-coupling between grains on magnetic properties of SrFe12O19 and $\alpha-\mathrm{Fe}_{2} \mathrm{O}_{3}$ composites," Mater. Res. Bull., vol. 57, pp. 13-18, 2014.

[32] A. D. Volodchenkov, S. Ramirez, R. Samnakay, R. Salgado, and Y. Kodera, "Magnetic and thermal transport properties of SrFe12O19 permanent magnets with anisotropic grain structure," Mater. Des., vol. 125, pp. 62-68, 2017. 